\title{
Décadrages Décadrages
}

cinéma, à travers champs Cinéma, à travers champs

4-5 | 2005

David Lynch

\section{Rhétorique et esthétique de la vidéosurveillance dans la culture contemporaine : Secrets for Sale (2003) d'Elodie Pong}

Mireille Berton

\section{CpenEdition}

Journals

Édition électronique

URL : https://journals.openedition.org/decadrages/616

DOI : $10.4000 /$ decadrages. 616

ISSN : 2297-5977

Éditeur

Association Décadrages

Édition imprimée

Date de publication : 10 avril 2005

Pagination : 135-146

ISBN : 978-2-9700582-1-2

ISSN : 2235-7823

Référence électronique

Mireille Berton, « Rhétorique et esthétique de la vidéosurveillance dans la culture contemporaine Secrets for Sale (2003) d'Elodie Pong ", Décadrages [En ligne], 4-5 | 2005, mis en ligne le 21 janvier 2013, consulté le 27 mars 2022. URL : http://journals.openedition.org/decadrages/616 ; DOI : https:// doi.org/10.4000/decadrages.616 


\title{
Rhétorique et esthétique de la vidéosurveillance
}

\author{
dans la culture contemporaine:
}

\section{Secrets for Sale (2003) d'Elodie Pong}

\author{
par Mireille Berton
}

"ADN/ARN (Any Deal Now / Any Reality Now): une installation interactive filmée où chaque visiteur était amené à confier puis vendre un secret personnel. Durant le parcours de l'installation (composé de 4 modules $\rightarrow$ salle d'attente, sas de préparation, studio, et cabinet), le visiteur/acteur répondait à un système sous l'œil attentif de 8 caméras. Arrivé à un certain point, il avait la possibilité (anonymement ou non) de vendre à Elodie Pong un secret personnel. Ce secret était enregistré sur un support audio-visuel (vidéo). Dans le dernier module, Pong intervenait en tant que performer, négociant le prix de cet achat dans la mesure où le secret l'intéressait, afin de l'ajouter à sa collection. Secrets for Sale est le film qui dévoile cette expérience".

Tel est le synopsis d'un moyen métrage constituant la phase 2 - succédant au projet ADN/ARN (phase 1), et précédant l'installation/performance I will not K.Y.S.S. (K-eep Y-our S-ecrets $S$-ecret) anymore (phase 3) - d'une recherche pluridisciplinaire menée sur le long terme (2001-2003) par la plasticienne américano-suisse Elodie Pong. Bien que son travail exigerait d'être analysé dans ses multiples facettes, je concentrerai ici mon propos sur la bande vidéo réalisée à partir de l'installation conçue dans le cadre du festival lausannois "Les Urbaines» (décembre 2001) et donnant lieu à une expérience qui se prolongera quatre mois durant lesquels quatre cents personnes s'engageront à vendre une part de leur intimité à l'artiste' 1 .

1 L'installation ADN/ARN sera reprise au Centre Culturel Suisse de Paris (printemps 2003) afin d'enrichir une collection qui totalisera 300 secrets contenus dans un film d'une durée totale de 9h30' (retenues sur 160 heures). Celui-ci a ensuite été diffusé au sein de l'exposition internationale "I Need You" tenue au Centre Pasquart de Bienne en Suisse (été 2004). Le film de 64 minutes, Secrets for Sale, continue pour sa part d'être projeté dans le cadre de nombreuses manifestations culturelles un peu partout dans le monde. 
2 Michel Foucault, Surveiller et punir. Naissance de la prison, Gallimard, Paris, 1975. Voir par exemple les études contenues dans CTRL Space. Rhetorics of Surveillance from Bentham to Big Brother, Ursula Frohne, Thomas Y. Levin et Peter Weibel (éd.), ZKM-Center for Art and Media/The MIT Press, Karlsruhe/Londres, 2002. 3 Tiré de la note d'intention d'Elodie Pong.
4 L'exhaustivité étant impossible face à la pluralité des approches et la diversité des œuvres ayant trait aux rapports entre art vidéo et surveillance, cet article se limite à soulever quelques questions et à évoquer quelques thèmes.

5 Tom Holert, "Die Kunst der Kontrolle. Anmerkungen zu Video, Authentizität und Überwachung", in Texte zur Kunst, ${ }^{\circ}$ 21, mars 1996, p. 69-79. La vidéo a également souvent été considérée comme un moyen facilitant l'expression de la subjectivité. C'est dans cette perspective qu'il faut comprendre l'idée qu'il s'agirait d'un médium essentiellement narcissique, comme le soutient Rosalind Krauss ("Video: An Aesthetic of Narcissism ", in Gregory Battock (éd.), New Artists Video: A Critical Anthology, Dutton, New York, 1979, p. 43-64).
Je commencerai d'abord par situer le film Secrets for Sale dans le cadre d'un phénomène culturel plus vaste, à savoir la réappropriation du dispositif de la vidéosurveillance par la "haute» culture (l'art contemporain) et surtout la "basse» culture (la télé-réalité) afin d'esquisser le champ dans lequel s'inscrit une œuvre qui travaille une série de thématiques (le contrôle, la pulsion scopique, la confession de soi, le marchandage de l'intimité, etc.) reprises, sur le plan théorique, par une réflexion référée bien souvent à l'épistémé panoptique de Michel Foucault ${ }^{2}$. Dans un deuxième temps, il s'agira d'étudier le statut particulier de ce «documentaire expérimental» $\mathbf{3}$ qui, en abordant les questions du travestissement, de la manipulation et de l'authenticité aussi bien au niveau du contenu que de la forme, pose du même coup le problème des conditions de vérité d'un discours vidéographique tiraillé par des exigences contradictoires.

\section{Le paradigme de la vidéosurveillance}

Les enjeux esthétiques, culturels et sociaux soulevés par la prolifération des différentes techniques de surveillance durant les dernières décennies du $\mathrm{XX}^{\mathrm{e}}$ siècle et au début du $\mathrm{XXI}^{\mathrm{e}}$ siècle, ont mobilisé autant des artistes que des penseurs de tous bords intéressés par l'analyse de l'image ou des mass media. Ce domaine étant amplement arpenté par des essais d'ordre "médiologique» ou philosophique (Michel Foucault, Gilles Deleuze, Régis Debray, Jean Baudrillard, Paul Virilio, Félix Guattari, Niklas Luhmann, Michel de Certeau, etc.), je me contenterai de souligner l'importante production d'œuvres et de textes prenant le pouls d'un paradigme au cœur de notre quotidien 4 . Qu'elle envisage les technologies de surveillance de manière globale, la vidéosurveillance en particulier ou encore l'art vidéo intégrant ce dispositif, cette pensée critique s'emploie souvent à avancer quelques hypothèses sur les spécificités d'une technique d'enregistrement ayant offert, dès son apparition, une alternative au cinéma. Ainsi, on rencontre souvent l'idée selon laquelle, jusque dans les années 1980, les images vidéo bénéficiaient d'une aura d'authenticité exploitée, par exemple, par une contre-culture désireuse de retrouver une virginité du regard sur le monde environnant, mais aussi d'interroger l'ambivalence d'un médium à la fois libérateur et coercitif 5 . Avec l'avènement de l'ère digitale, toutefois, les paramètres habituellement usités pour tenter de cerner l'«ontologie» de l'image indicielle (photographie, cinéma, vidéo) ont dû se soumettre à des processus de révision.

En effet, la possibilité de coupler les techniques analogiques à des logiciels informatiques traitant l'image numériquement fait basculer la 
vidéo (autant que le cinéma et la photo) dans le règne des «images de surface» 6 où les notions d'apparence, de superficialité et d'inauthenticité entraînent une certaine forme de désincarnation de la représentation. Car «l'opération numérique procède objectivement à un déracinement, à une sorte de coupure ombilicale projetant la forme-image dans un mouvement de déterritorialisation sans précédent» $\mathbf{7}$ qui arracherait ainsi l'image à sa matrice sise dans le réel. Comme le suggère Thomas Y. Levin, les nouveaux médias périment la question de la véracité de l'image, de son «réalisme» photographique - et donc du "ça a été» de Roland Barthes - pour générer de nouvelles modalités de représentation audiovisuelle, dont le paradigme de la surveillance constitue un cas de figure possible ${ }^{\mathbf{8}}$. Si l'analyse des fonctions rhétoriques de la surveillance menée par Levin se limite aux films contemporains, on peut l'extrapoler sans difficulté à l'art vidéo qui, très rapidement, s'est emparé de ce «penchant naturel» de la vidéo pour l'observation et le quadrillage d'un espace particulier dans un temps continu, et surtout pour la transmission d'images en temps réel.

Des artistes comme Bruce Nauman (Video Surveillance Piece: Public Room, Private Room, 1969-1970), Dan Graham (Video Monitor on Time Delay Room, 1974), Julia Sher (Productive Engineering, 1993; Security Land, 1995) 9, Frank Thiel (City TV Berlin, 1997-1999), Sophie Calle (Unfinished, 2003), parmi beaucoup d'autres, ont proposé leur propre lecture de la vidéosurveillance et de ses corollaires. Basée sur une série de composantes structurelles et formelles - hiérarchisation des espaces, des regards et des pouvoirs; aporie d'une visibilité totale; indifférence de l'œil-caméra; "esthétique de l'ennui» $\mathbf{1 0}$; etc. - la vidéosurveillance ne se contente pas d'envahir les espaces publics, mais continue d'occuper l'aire de la culture contemporaine qui ne cesse d'interroger ses fondements idéologiques, esthétiques et sociaux ${ }^{\mathbf{1 1}}$.

Alors que "la métaphysique de la trace» $\mathbf{1 2}$ de l'image photo-cinématographique traverse une zone de turbulences sans précédent, le paradigme de la surveillance semble ouvrir, à suivre Levin, une voie inédite aux pratiques audiovisuelles. Il permet notamment de déplacer le «souci de l'analogon»13 - c'est-à-dire de la conformité de l'image au réel - vers la question de la diffusion en temps réel d'un fragment de réalité en action. Partageant avec la télévision ce statut d'«énoncé indexique» défini comme un discours "fondé à pouvoir dire quelque chose de la réalité» $\mathbf{1 4}$ - discours d'autant plus convaincant que celle-ci est prise et (idéalement) restituée sur le vif -, la séquence de vidéosurveillance, à l'instar du direct télévisuel, s'offre au spectateur comme un lieu riche en promesses événementielles, comme «un terrain privilégié à
6 Christine Ross, Images de surfaces: l'art vidéo reconsidéré, Artexte, Montréal, 1996.

7 Alain Renaud-Alain, "L'imaginaire numérique", in Jean-Michel Frodon, Marc Nicolas, Serge Toubiana (éd.), Le cinéma vers son deuxième siècle, Editions Le Monde, Paris, 1995, p. 61.

8 Thomas Y. Levin, "Rhetoric of the Temporal Index: Surveillant Narration and the Cinema of 'Real Time, ", in CTRL Space. Rhetorics of Surveillance from Bentham to Big Brother, op. cit., p. 578-593.

9 Security Land se présente comme un site Internet interactif où le visiteur doit remplir un questionnaire visant à estimer son degré de volonté à participer à un projet qui tend vers l'exploration de l'intimité des gens. Le parallèle avec ADN/ARN est ici tout à fait frappant. Site Internet: http://adaweb.com. Cf. "Danger Dirty Data. A Project for Artforum by Julia Sher with an Introduction by Andrew Hultkrans", in Artforum, septembre 1995, p. 74-77.

10 Nicolas Thély, vu à la webcam (Essai sur la web intimité), Les Presses du Réel, Paris, 2002.

11 Sur ces questions, voir le dossier "Vidéosurveillance et fiction " dirigé par Christophe Kihm pour Artpress, $n^{\circ}$ 303, juillet-août 2004.

12 Laurent Jullier, "La métaphysique de la trace et sa crise actuelle", in Champs visuels, n० 1 ("Réalités de I'Image. Images de la réalité»), mars 1996, L'Harmattan, p. 114-120.

13 Jean-François Diana, "La valeur de réel à la télévision", in Champs visuels, n 1 , op. cit., p. 100.

14 Jean-Pierre Esquenazi, "Qu'est-ce qu'un discours 'vrai'? L'énoncé indexique", in Champs visuels, $n^{\circ} 1$, op. cit., p. 9. Plus précisément, les énoncés indexiques proposent des "assertions qui s'exposent explicitement à un jugement, qui les déclarera vraies ou fausses en fonction de leur correspondance avec des faits concrets". Voir aussi, "Qu'est-ce qu'un discours 'vrai' ? (2) L'image "vraie, aujourd'hui ", in Champs visuels, $n^{\circ} 2$ ("Réalités de I'Image. Images de la réalité (2)"), juin 1996, L'Harmattan, p. 9-18. 
15 Jean-François Diana, "La valeur de réel à la télévision", op. cit., p. 101.

16 Cf. Alain Ehrenberg, "La vie en direct ou les shows de l'authenticité", in Esprit, n 1, 1993, p. 13-35.

17 Philippe Quéau distingue les "régimes iconiques" (comme la peinture, la photographie, le cinéma, la télévision et la vidéo) des "régimes idoliques" des images de synthèse, des simulateurs et de la robotique (car "c'est notre pensée que nous idolâtrons "à travers eux, p. 254.). Cf. Philippe Quéau, Eloge de la simulation. De la vie des langages à la synthèse des images, INA, Paris, 1986.

18 Voir à ce sujet, Mary Ann Doane, "Information, Crisis, Catastrophe", in Patricia Mellecamp (éd.), Logics of Television, Indiana UP, Bloomington, 1990, p. 222-239.

19 Comme le remarque David Rodowick, les nouveaux médias ne remettent pas fondamentalement en cause le réalisme de l'image traditionnelle, ainsi que le phénomène d'impression de réalité qu'elle engendre, mais au contraire y contribuent indirectement et avec une efficacité bien comprise par l'industrie du cinéma hollywoodien. En effet, "dans le conflit avec le 'digital, se réaffirme la valeur esthétique des images analogiques: elles sont plus r réelles, que les simulations digitales, pas seulement au cinéma mais aussi dans les jeux et autres formes de divertissements électroniques". David Rodowick, "La Vie virtuelle du film", in Cinergon, no 15 ("Où va le cinéma"), 2003, p. 20 (texte paru dans Publication of the Modern Language Association, octobre 2001, p. 1396-1404, traduit de l'anglais par Maxime Scheinfeigel).

20 II s'agit, pour le dire rapidement, de la connaissance immédiate que le spectateur peut avoir face à une image indicielle dont l'origine (production mécanisée d'une image comme empreinte chimique du réel) ne fait aucun doute et s'impose en quelque sorte d'ellemême. Jean-Marie Schaeffer, L'image précaire, Seuil, Paris, 1987.

21 Cf. Doug Hall et Sally Jo Fifer (éd.), Illuminating Video. An Essential Guide to Video Art, Aperture/BACV, New York, 1990. Pour les rapports entre vidéo et télévision voir les études récentes de Raymond Bellour (L'entre-images: photo, cinéma, vidéo, Editions de La Différence, Paris, 1990; L'entre-images 2: mots, images, P.O.L, Paris, 1999).

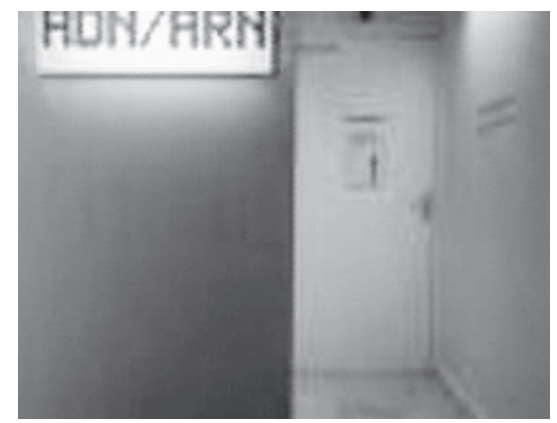

ENTRÉE

l'émergence» d'un "instant-réel»15. Certes, la télé-réalité naissante avait déjà destitué le vraisemblable au profit de l'authentique, mais l'image de vidéosurveillance semble repousser encore plus loin l'urgence d'une capture à l'état brut d'une réalité vécue ${ }^{\mathbf{1 6}}$, posant ainsi le problème de la mimesis avec une acuité et en des termes inédits.

L'image électronique (vidéo, télévision) de la surveillance, tout en participant au même régime iconique que la photographie ou le cinéma, construit un autre rapport à l'espace, mais surtout une autre relation au temps qui devient un temps de l'actualité17. Forte de cette impression d'instantanéité (et d'ubiquité) produite sur le spectateur, cette imagerie va permettre de "réinjecter» dans la représentation une dose d'indicialité contribuant à cimenter le contrat de croyance passé avec celui-là. D’une part, le recours - typiquement télévisuel mais aussi cybernétique - à ce stratagème de l'image diffusée en temps réel concourt à détacher la représentation de son adhérence (trop stricte) aux catégories de l'espace pour l'ancrer dans le primat de la temporalité18. D'autre part, l'emploi $\mathrm{du}$ vocabulaire technique et visuel fourni par la vidéosurveillance fonctionne comme le garant d'une illusion de réalité redéfinie par les nouvelles technologies $\mathbf{1 9}$, remplaçant, pour ainsi dire, le rôle joué par le «savoir de l'arché» $\mathbf{2 0}$ attaché à l'image «classique» qui permet d'attester de l'adéquation à son référent.

\section{Télé-réalité et surveillance: connivences}

Il existe de nombreux points de convergences entre l'histoire de la vidéo, de la vidéosurveillance et celle de la télévision qui ont, par ailleurs, fréquemment été investigués par des artistes vidéo soucieux de dénoncer, par exemple, les effets pervers de ces dispositifs coupables d'aliéner l'individu, d'exercer une hégémonie idéologique ou un contrôle massif sur l'information 21 . A l'inverse, les jeux de télé-réalité, au lieu d'en dresser 


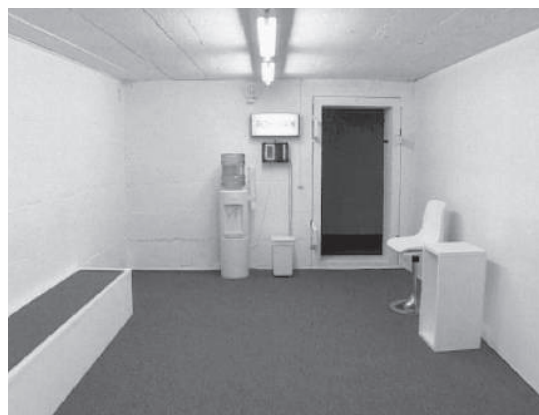

SALLE D'ATTENTE

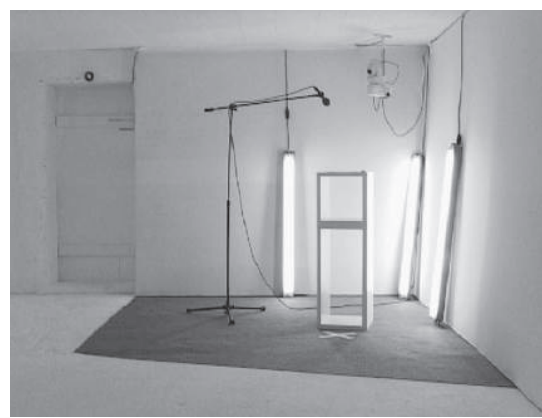

SAS DE PRÉPARATION

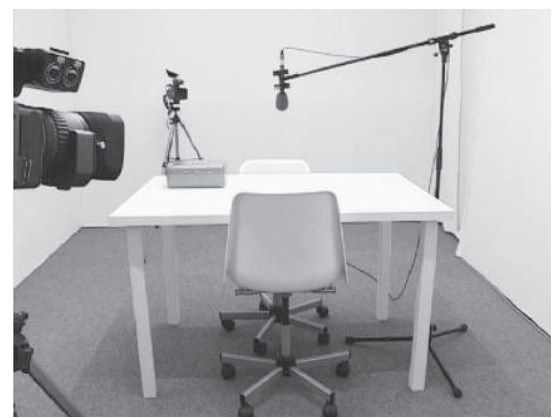

CABINET une critique, représentent l'expression la plus achevée d'une synthèse harmonieuse entre la société de contrôle décrite par Michel Foucault et Gilles Deleuze 22 et l'institution télévisuelle. Si l'art et le cinéma contemporains ont exploité largement cette thématique de la vidéosurveillance ${ }^{23}$, la télé-réalité, en suivant l'exemple de la culture "webcam ", l'a érigée au rang de principe structurel et formel $\mathbf{2 4}$.

De la télé-vérité dont la vocation consistait à révéler la vie de Monsieur-tout-le-monde dans les années 1950, on est passé à une téléréalité animée par une "prétention à parler au nom du réel» $\mathbf{2 5}$ et en temps réel du quotidien de gens ordinaires $\mathbf{2 6}$. Pour cela, les producteurs équipent des lofts de caméras de surveillance quadrillant l'espace, à l'affût d'un incident qui pourrait faire saillie - donc faire spectacle - dans un flux d'images réglé par «le présent continu de la transmission» $\mathbf{2 7}$. Telles sont les limites d'une observation qui avance «à l'aveuglette» dans un champ ouvert à de potentielles péripéties qui fourniraient spontanément la matière première d'un récit reposant, cependant, sur le "paradigme de la mise en scène» $\mathbf{2 8}$. Car la télévision récupère volontiers des images de vidéosurveillance, mais à condition d'en expurger le caractère foncièrement itératif et monotone pour ne retenir que les instants prégnants, c'est-à-dire les «débordements imprévus » 29 d'un système conçu pour pratiquer une maîtrise absolue du perçu. La phénoménologie de l'image de vidéosurveillance est donc double puisqu'elle se déploie à la fois dans l'exercice contraignant d'une vigilance ininterrompue et dans le prélèvement ponctuel, à l'intérieur d'une étendue (spatio)temporelle, de moments particulièrement signifiants du point de vue d'une dramaturgie non concertée.

Récupérant ce statut ambivalent de l'image de vidéosurveillance, la télé-réalité va susciter une série de discussions sur le régime de crédibilité qui sous-tend la création et la réception de ces nouvelles formes de
22 Cf. Gille Deleuze et Félix Guattari, Mille Plateaux, Paris, Editions de Minuit, 1980; Gilles Deleuze, "Post-scriptum sur les sociétés de contrôle" [1990], Pourparlers, 1972-1980, Minuit, Paris, 1990, p. 240-247. Dans le second texte, Deleuze note que les sociétés disciplinaires analysées par Michel Foucault ont cédé la place, à la fin du XX siècle, aux sociétés de contrôles fondées sur la prééminence de l'argent, du chiffre, du marketing, de l'ordinateur, etc., l'homme endetté ayant ainsi remplacé l'homme enfermé.

23 Parmi les films citons, entre autres, Sliver (Philip Noyce, 1993); Wag the Dog (Barry Levinson, 1997); Snake Eyes (Brian De Palma, 1998); The Truman Show (Peter Weir, 1998); le remake de The Conversation (1974) de Francis F. Coppola, Enemy of State (Tony Scott, 1998); Time Code (Mike Figgis, 2000). En Suisse, le film vidéo de Pierre-Yves Borgeaud et de Stéphane Blok, iXième. Journal d'un prisonnier (2003) a fait un usage original de la vidéosurveillance.

24 Voir à ce sujet mon article dans le no 1-2 de Décadrages, automne 2003, "Le regard et le miroir dans Loft Story. Lecture d'un dispositif de télé-réalité à la lumière de Michel Foucault et Jacques Lacan".

25 François Jost, La télévision du quotidien. Entre réalité et fiction, De Boeck Université, Bruxelles, 2001, p. 7.

26 Cf. "De la télé-vérité aux reality shows", Dossiers de l'audiovisuel, n 53, 1994.

27 Christophe Kinm, "Vidéosurveillance, regard et identité. Les modalités de la présence", in Artpress, op. cit., p. 28.

28 Patrice Blouin, "Le miroir indifférent. Vidéosurveillance et mise en scène", in Artpress, op. cit., p. 34.

29 lbid., p. 35. 
30 François Jost, op. cit.

31 Ceux-ci apparaissent par exemple à travers le "jeu" des "visiteurs/acteurs" qui adoptent certaines postures corporelles ou mentales vis-à-vis d'une caméra encourageant à endosser un certain rôle, qu'il soit familier ou non à l'intéressé.

32 Note d'intention d'Elodie Pong. divertissement. Comme le note François Jost ${ }^{\mathbf{3 0}}$, si le débat sur la restitution de la réalité par les médias audiovisuels connaît une longue histoire, dans le cas de la télé-réalité nous sommes confrontés à un phénomène qui, en oblitérant la distinction entre fiction et réalité, nous oblige à repenser les questions du vrai/faux, vraisemblable/invraisemblable, possible/impossible, probable/improbable, etc. Que l'on envisage les séquences visionnables en (pseudo-)direct sur le Net ou les résumés quotidiens diffusés par ondes hertziennes, le spectateur consomme des images qui s'insèrent au carrefour de trois principaux genres télévisuels définis par Jost comme étant le "mode fictif» (film, téléfilm, série), le "mode ludique» (variétés, jeux) et le "mode authentifiant» (journaux télévisés, débats en direct).

Bien que la télé-réalité entrecroise ces trois genres, elle paraît s'articuler de manière plus ferme au mode authentifiant qui caractérise les émissions délivrant au téléspectateur des faits et des paroles non truquées, même si cette "réalité» est le fruit d'une élaboration minutieuse. Dans le cadre d'une tendance généralisée de la télévision à superposer les temporalités (vrai direct, faux direct, direct différé, etc.), l'imaginaire de la vidéosurveillance génère le fantasme perceptif d'une saisie directe de l'instant présent, procurant ainsi une illusion de réalité qui semble primer sur tous les indices de facticité inscrits dans la représentation $\mathbf{3 1}$. La rhétorique et l'esthétique de la «télé-surveillance» apparaissent ainsi comme décuplant la capacité d'enregistrement-restitution d'une réalité objective prise sur le vif, capacité propre à un médium - la télévision bien plus indiciel que le cinéma, et plus encore que la photographie.

\section{Secrets for Sale à l'aune de la télé-réalité}

Le dispositif de caméra-surveillance à l'œuvre dans le film Secrets for Sale présente, au premier abord, bien des analogies avec ceux habituellement conçus par la télé-réalité. Le candidat au système ADN/ARN commence par céder les droits de sa propre image. Il traverse ensuite des espaces qui n'échappent pas à «l'œil attentif de 8 caméras» 32. Cet espace est lui-même subdivisé en modules de deux types qui instaurent un rapport différencié du visiteur/acteur avec la caméra: il y a, d'un côté, des modules panoptiques invitant le sujet à ignorer la présence des objectifs destinés à capturer une phénoménologie du corps (et du comportement) dans sa naturalité; il y a, de l'autre côté, des modules-confession qui provoquent un face-à-face entre le dispositif de captation et la personne filmée. Les caméras sont donc investies d'une double fonction, instituant tantôt le tabou du regard-caméra, appelant tantôt le regard dialogique. Le parcours dans le système est partiellement contrôlé par 
une voix off qui se confond avec l'instance de production-énonciation. Celle-ci cherche à obtenir des candidats une partie de leur intimité livrée sous la forme d'un secret qui, une fois formulé, passe du domaine privé au domaine public. Ceux qui refusent de se prêter au jeu sont priés de garder le secret (précisément) quant au dispositif. Les séances de confidences créent un «espace imaginaire de relation" $\mathbf{3 3}$ constitué symétriquement comme une interface liant un espace réel (le studio d'enregistrement) à un espace virtuel (l'espace des spectateurs). Le régime phatique régissant le fonctionnement de cette interface produit une esthétique de la présentation $\mathbf{3 4}$ basée sur une relative fixité et frontalité du cadre. De plus, les signes d'auto-réflexivité sont omniprésents: en sus des regards-caméra, on nous montre des plans sur des caméras qui panoramiquent, sur des micros et autres appareils de prise de vue, des zooms appuyés, etc. Le discours entourant l'installation/performance rappelle celui du Loft 35 : il est question d'une "expérience» (ou d'une «aventure») - notion clé de la télé-réalité - qui fascine les "candidats» qui la trouvent "intéressante", mais qui inquiète ceux qui accusent un «système fascisant» ressenti comme "manipulateur».

Cependant, ces correspondances, aisées à établir, n'épuisent pas totalement l'analyse d'un dispositif artistique singulièrement en phase avec son temps, et notamment avec une forme de récréation télévisuelle fortement débattue, parfois sur le mode de la controverse, par des intellectuels de tous bords. Il semblerait que, tout en reprenant certains principes essentiels de la télé-réalité, ADN/ARN opère sur celle-ci une série de renversements qui mettent le doigt sur l'envers du décor. En effet,
33 Noël Nel, "Les régimes scopiques. Essai de typologie (1)", in Champs visuels, $n^{\circ} 1$, op. cit., p. 54.

34 Cf. Noël Nel, Le débat télévisé, Armand Colin, Paris, 1990.

35 Cf. Peter Weibel "Pleasure and Panoptic Principle", in CTRL Space. Rhetorics of Surveillance from Bentham to Big Brother, op. cit., p. 207-223.

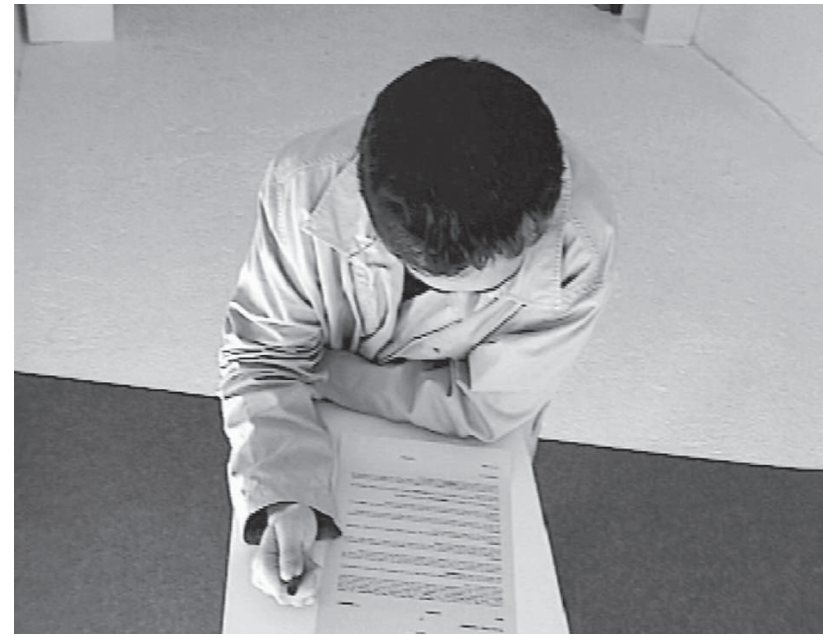

LA SIGNATURE DU CONTRAT 

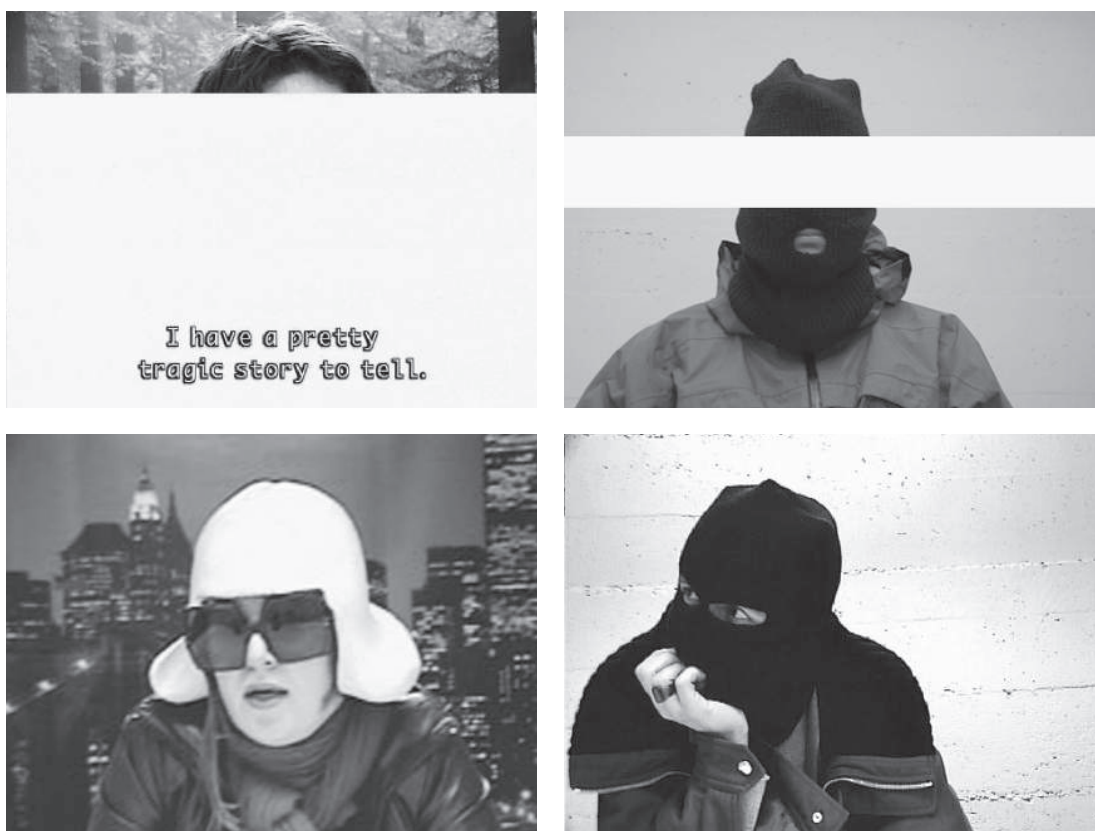

si les productions des grandes chaînes de télévision se réclament d'une honnêteté et d'une transparence absolues, beaucoup d'éléments restent dissimulés à la vue et au savoir du téléspectateur. A certains égards, l'installation (et donc aussi le film vidéo) permet de démasquer la logique à la fois mercantile, répressive et factice qui gouverne ces programmes. En montrant quelques «ficelles» indispensables (mais médiatiquement gênantes) au bon fonctionnement de ces appareillages ludiques, elle lève le voile qui contribue parfois à anesthésier l'esprit critique du téléspectateur d'une émission comme Loft Story.

Secrets for Sale, effectivement, commence par exhiber le processus de sélection fondé sur la volonté de participation du sujet à un projet qui préserve une part de mystère, même après la signature du contrat. Il faut donc des candidats coopératifs, disposés à prendre des risques, à livrer une fraction d'eux-mêmes dont la valeur marchande sera jaugée à l'issue de l'itinéraire. Les phases d'entrée (intégration au système via une adhésion écrite aux règles du deal) et de sortie (offre de compensation financière après satisfaction du "client») apparaissent sans détour dans le film. Après avoir manifesté leur désir de progresser dans l'installation, les visiteurs s'avèrent contraints de répéter des phrases dictées par la voix off masculine qui les accueille dans le premier module, abdiquant ainsi symboliquement, non seulement l'accès à leur image, mais aussi 
leur liberté de pensée et de parole. Le simulacre s'affiche, quant à lui, à travers la série d'accessoires (postiches, perruques, masques, etc.) et de procédés techniques (voix déformée électroniquement, bande blanche horizontale, floutage, etc.) destinés à travestir l'identité du candidat qui désire conserver l'anonymat. Il est également assumé par les participants eux-mêmes qui jouent volontairement avec le dispositif - en refusant de jouer le jeu justement - ou avec Elodie Pong elle-même qui dote le système d'une présence (et d'une voix) humaine incarnant l'autre pôle interactif.

Alors que les programmes de télé-réalité, tout à la fois, gardent une certaine discrétion sur les compensations financières accordées aux non-gagnants, déguisent la simulation sous la forme d'une fête perpétuelle, censurent les opinions contraires et se débarrassent des individus rétifs, Secrets for Sale arbore clairement cette série de mécanismes qui doivent habituellement rester dans l'ombre pour préserver la viabilité de ces dispositifs. Il offrirait ainsi une version plus sincère d'une téléréalité pour laquelle l'autorité d'une observation totale dépend du partage entre des zones de visibilité et d'invisibilité. Si la télé-réalité transforme toute réalité en image, c'est toujours au prix d'une opacité nécessaire à la survie de tels programmes enclins à la dérobade et à la «feintise» $\mathbf{3 6}$.

\section{Le jeu de la vérité}

Toutefois, Secrets for Sale (autant que le système ADN/ARN) n'est pas exempt d'un certain nombre de contradictions reflétées, entre autres,

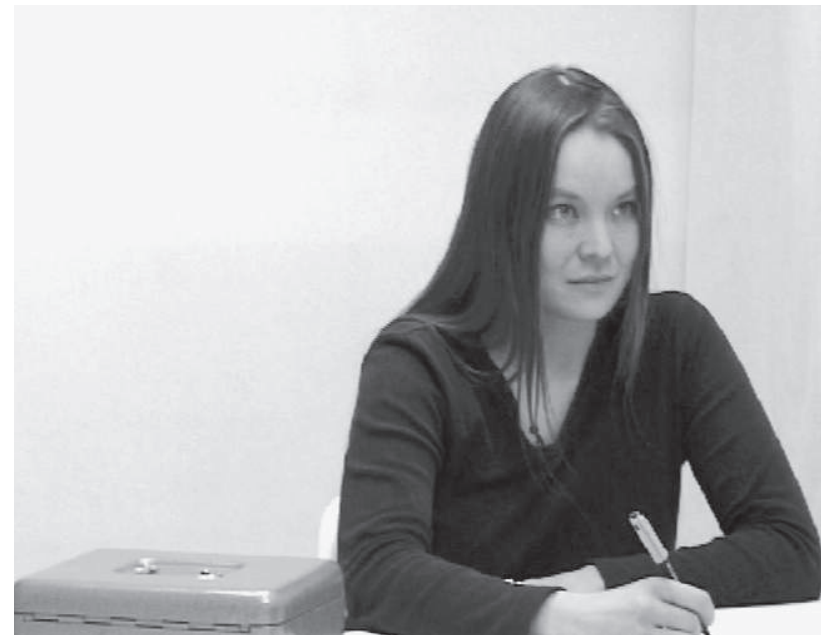

ELODIE PONG
36 La feintise concerne tous les discours qui parlent au nom d'une vérité cependant construite de toutes pièces. II s'agit donc de singer une réalité afin de produire un effet de vérité. François Jost, L'empire du loft, La Dispute, Paris, 2002. Voir aussi, François Jost, "Le feint du monde", in La communication de l'information, L'Harmattan, Paris, 1996-1997. 
37 Tiré de la note du producteur, Thierry Spicher (je souligne).

$38 \mathrm{~A}$ une époque où rien n'est plus vraiment considéré comme tabou, comment interpréter ces "secrets" qui révèlent, certes, quelque chose sur les individus qui en sont porteurs, mais pas forcément au niveau du contenu luimême? En effet, plus que le secret en soi, c'est le choix de raconter telle ou telle anecdote, plutôt qu'une autre, qui est susceptible de trahir une parcelle du profil psychologique du candidat.

39 Thierry Spicher (je souligne).

$\mathbf{4 0}$ La notion de radiographie, puisée dans le champ lexicologique de la médecine, renvoie bien à l'idée d'une expérience de laboratoire (voir la blancheur des murs et l'éclairage artificiel) employant l'individu comme un "cobaye", tel que le dénonce une voix féminine à un moment donné du film où le jeu est mis en suspens, pour céder la place à un dialogue off entre cette visiteuse explicitant son malaise face à un dispositif ressenti comme "fascisant" et Elodie qui semble déroutée par une telle vision de son projet, pourtant pertinente.

41 Note d'intention d'Elodie Pong. Je souligne. A titre de rappel, les émissions de télé-réalité du type Big Brother se targuent souvent d'avoir choisi un panel de gens représentatifs d'une diversité socioculturelle. Ici, il s'agit plutôt de présenter un échantillon des tourments psychologiques de la société actuelle.

42 Plusieurs candidats tentent d'établir un contact plus personnel avec la performer qui, elle, s'efforce toutefois de garder une certaine distance, alors qu'elle semble parfois les connaître personnellement. On sent de manière générale que les participants ont envie d'engager le dialogue avec elle afin de connaître son point de vue sur le concept qui guide son travail.

43 Sur le transfert de la fonction de surveillance divine aux technologies panoptiques modernes, cf. Astrit Schmidt-Burkhardt, "The All-Seer: God's Eye as Proto-Surveillance", in CTRL Space. Rhetorics of Surveillance from Bentham to Big Brother, op. cit., p. 17-31. dans les propos de son producteur, Thierry Spicher, qui le présente comme "un film, un document, un documentaire, une fiction, une vidéo d'art et le titre d'un film présenté en installation». Il continue en précisant que "tout cela est vrai simultanément, ou plutôt tout cela est vrai ou faux selon l'horizon d'attente généré chez le spectateur, ou encore selon le mode de présentation choisi...»37. Il ne s'agit pas ici de trancher dans la pluralité des propositions avancées par Spicher, mais de mettre en exergue, non seulement les notions de fiction et de documentaire, mais encore l'une des questions attachées au paradigme de la surveillance évoqué plus haut, à savoir celle de la vérité et de l'authenticité. Si le maintien d'une certaine indécidabilité entre points de vue fictionnalisant et doumentarisant s'avère salutaire, la récurrence de références à une représentation fidèle de la réalité comme gage de vérité du discours vidéographique vient compliquer passablement la donne.

Le titre de l'installation/performance - en français : «Tout deal, toute réalité, maintenant» - nous place d'emblée sur la piste d'une recherche d'authenticité nichée dans la réalité elle-même, et plus précisément dans les secrets 38 révélés par les candidats. C'est cette collection amassée au fil des rencontres qui permettra de déboucher sur la «construction d'un document» $\mathbf{3 9}$ fonctionnant comme une "troublante radiographie $\mathbf{4 0}$ d'une époque où tout est à vendre» $\mathbf{4 1}$. On aspire donc à une logique de transparence et d'hyperlucidité, aussi bien au niveau psychologique des liens intersubjectifs $\mathbf{4 2}$, qu'au niveau technologique des "yeux électroniques » des caméras de surveillance douées d'une omni-voyance autrefois attribuée à l'œil divin 43. Même si le système connaît parfois des courtscircuits, idéalement, c'est le maintien d'une "vérité» de l'expérience (mais aussi d'une sincérité réciproque) qui est convoitée par les deux parties du deal, et plus spécialement par la performer qui, à plusieurs reprises, prend soin de s'assurer de la véracité du secret de son candidat. Il n'est pas étonnant de constater que cette préoccupation concerne tout

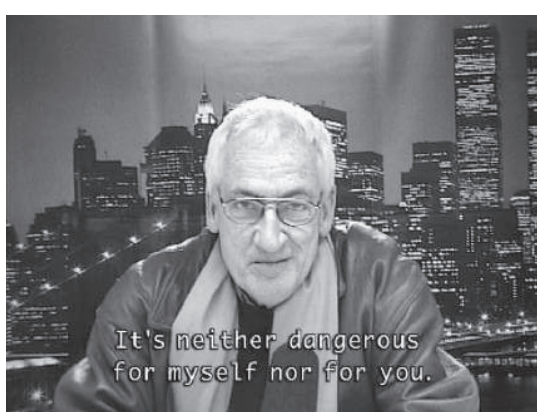


particulièrement les secrets qui ont trait à l'intimité, comme le garçon qui avoue se masturber avec ses excréments $\mathbf{4 4}$.

Comment comprendre, dès lors, ce souci de concilier, d'une part, une réaction "[authentique] face à des propos souvent surprenants $\mathbf{4 5}$, et, d'autre part, la reconnaissance d'une relativité des propos des candidats en termes de vérité ? $\mathbf{4 6}$ Comment comprendre la cohabitation, au sein d'un même projet, entre deux exigences paradoxales (tolérer le mensonge, tout en vérifiant la véracité des secrets) ? C'est comme si la nature même de ce dispositif panoptique inférait, en vertu de ses diverses propriétés (omnivoyance, contrôle, transparence, aveu, pouvoir, etc.), le déploiement d'un discours vériste, parfois en dépit de la volonté des uns et des autres $\mathbf{4 7}$. Pris au piège d'une machinerie dont les effets encouragent l'émergence d'une injonction tacite au respect d'une vérité vraie, la "présentation de soi et l'engagement à jouer sa présence dans un dispositif de vidéosurveillance $\mathbf{4 8}$ entraînent un besoin de jouer à être soi-même (par ailleurs, leitmotiv des candidats du Loft), à jouer une partie de soi-même dans une partie qui ressemble au jeu de la vérité.

Ce principe de toute-puissance du discours authentifiant se vérifie aussi bien dans l'installation que dans la bande vidéo elle-même: alors que les individus intégrés au système ont assimilé l'autorité du regard disciplinaire (Michel Foucault), les obligeant à jouer franc jeu (et à être sages comme des "images» de télé-réalité, c'est-à-dire pittoresques et touchantes), le film (composé d'images de vidéosurveillance) reprend à son compte certains traits stylistiques du reportage télévisuel annexé plus haut aux énoncés indexiques. En effet, Secrets for Sale peut être envisagé comme un reportage sur l'installation ADN/ARN qui en retracerait les moments forts, la médiation audiovisuelle visant la restitution vraie d'un événement réel, et ceci en soulignant l'existence des faits montrés à l'image par quelques procédés authentifiants souvent utilisés à la télévision: un panel restreint de cadrages, une limitation de l'espace représenté, la présence d'une instance d'énonciation (là le journaliste, ici les candidats ou Elodie Pong), un son direct, des «mouvements de caméra consciencieusement approximatifs" (zooms maladroits), l'exhibition du dispositif technique (affectée là, voulue ici), les regards-caméra 49.

Ainsi, le discours vériste semble contaminer tous les éléments constitutifs du film, à commencer par l'usage d'images de vidéosurveillance appréhendées ici dans leur fonction première d'observation d'une «intrusion hasardeuse de l'indice de réel» $\mathbf{5 0}$. Cependant, en dépit d'une parenté frappante du film avec le langage de la vidéo/télé-vision, son statut effectif reste problématique car il condense des messages contradictoires qui rendent difficile le cheminement interprétatif. On peut,
44 II y aurait lieu de développer cette question de l'intimité - qui forme par ailleurs un des axes majeurs du travail d'Elodie Pong - pour montrer son imbrication avec celle de l'identité (voir le titre ADN/ARN), mais également pour tirer un parallèle avec la télé-réalité friande d'exposer les affres de l'intimité des personnes qui sont volontaires à l'enfermement.

45 Thierry Spicher.

46 Elodie Pong estime que ces "confessions ambiguës", ces "silences retenus" ou ces "logorrhées impudiques" proviennent "de personnes qui jouaient sincèrement ou non le jeu".

47 Dans un tout autre contexte, François Albera parle d'“inférence de l'outil" à propos de la vidéo dont les propriétés techniques favorisent une appréhension narcissique du médium. François Albera, "Cinéma/Vidéo: corps à corps", in $7^{\text {e }}$ Semaine Internationale de Vidéo *(Biennale de l'Image en Mouvement), SaintGervais, Genève, 1997, p. 104.

48 Christophe Kihm, "Vidéosurveillance, regard et identité. Les modalités de la présence", in Artpress, op. cit., p. 29.

49 Je reprends ici, en les adaptant, les propos de Jean-François Diana, "La valeur de réel à la télévision", op. cit., p. 103.

50 lbid., p. 105. 
51 On rencontre une occurrence supplémentaire de ce type de contradictions à un niveau plus général, notamment dans le traitement du présupposé vériste sous-tendant le dispositif panoptique qui oscille entre absence de mise en perspective de la part de l'espace de production et stratégies inventives de détournement de la part des visiteurs/participants.

52 Michel Foucault, Les mots et les choses, Gallimard, Paris, 1966, p. 331. certes, trouver certaines traces d'une «écriture cinématographique» dont parle Spicher, comme, par exemple, du côté de l'espace de lecture où l'idiome esthétique de la vidéosurveillance incite le spectateur à entretenir avec le perçu un rapport fondé sur l'expectative d'un pic narratif. Mais l'ambiguïté entre l'«hyperindicialité» temporelle de l'image vidéo - qui rapproche le film de la télévision - et la tentative d'articuler ces fragments de vidéosurveillance en une structure narrative cohérente - qui l'apparente au cinéma -, ne fait que dérouter davantage le spectateur ou l'analyste $\mathbf{5 1}$. A force de conjuguer simultanément les codes de l'art vidéo, du cinéma documentaire, du cinéma de fiction et de la télévision, Secrets for Sale finit pas en perdre son latin et à n'appartenir à aucun d'entre eux. D'où la multivalence d'un objet qui tente de dire «la vérité des choses " à travers "une vérité qui est de l'ordre du discours »52, d'un discours croisant des modalités esthétiques et narratives hétérogènes. Par son inscription dans une culture visuelle encline aux métissages sémiotiques et formels, Secrets for Sale constitue ainsi l'occasion de saisir une partie des enjeux esthétiques et théoriques qui fondent le débat actuel sur le phénomène multimédiatique. 